\title{
PROCESS AND SYSTEMS The contribution of human factors and ergonomics to the design and delivery of safe future healthcare
}

\author{
Authors: Mark Sujan, ${ }^{\mathrm{A}}$ Laura Pickup, ${ }^{\mathrm{B}}$ Paul Bowie, ${ }^{\mathrm{C}}$ Sue Hignett, ${ }^{\mathrm{D}}$ Fran Ives, ${ }^{\mathrm{E}}$ Helen Vosper ${ }^{\mathrm{F}}$ and Noorzaman Rashid ${ }^{\mathrm{G}}$
}

Human factors and ergonomics (HF/E) is concerned with the design of work and work systems. There is an increasing appreciation of the value that HF/E can bring to enhancing the quality and safety of care, but the professionalisation of HF/E in healthcare is still in its infancy. In this paper, we set out a vision for HF/E in healthcare based on the work of the Chartered Institute of Ergonomics and Human Factors (CIEHF), which is the professional body for HF/E in the UK. We consider the contribution of HF/E in design, in digital transformation, in organisational learning and during COVID-19.

KEYWORDS: ergonomics, human factors, patient safety

DOI: 10.7861/fhj.2021-0112

\section{Introduction}

Future healthcare faces significant challenges, including complex care needs of an increasingly elderly population, staff shortages and burnout, and global crises (such as the COVID-19 pandemic). Responding to these challenges and providing safe care requires not only improvements in medicine, but also changes to how we work (ie how health and social care are designed and delivered). Healthcare providers need to communicate, coordinate and collaborate better across professional, departmental and organisational boundaries. Digital technologies (such as novel artificial intelligence (AI) applications) need to work well with people in order to harness their full potential. Health systems and organisations need to be agile and able to adapt to changing circumstances and demands. The design of work and work systems is the focus of

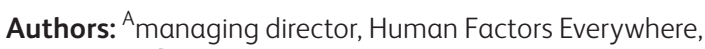
Woking, UK; ${ }^{\mathrm{B}}$ managing director, LP Human Factors, Taunton, UK; ${ }^{C}$ programme director, NHS Education for Scotland, Glasgow,

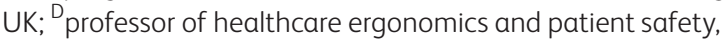

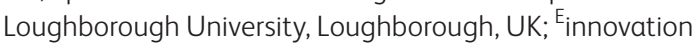
manager, West Midlands Academic Health Science Network, Edgbaston, UK; F senior lecturer, Robert Gordon University, Aberdeen, UK; ${ }^{G}$ chief executive, Chartered Institute of Ergonomics and Human Factors, Wootton Wawen, UK human factors and ergonomics (HF/E).

$\mathrm{HF} / \mathrm{E}$ is a scientific discipline that takes a systems perspective, and aims to design interactions between people and other elements of the system to optimise human wellbeing and overall system performance. ${ }^{1}$ Aspects of HF/E have been used to improve patient safety and the quality of care for over 20 years, but frequently with a narrow focus on teamwork and non-technical skills. ${ }^{2}$ While such interventions can be valuable, they are best thought of as one element of the HF/E toolbox, which should be accompanied by consideration of how the wider work system can be designed. This includes, for example, the design of tools and equipment, task design, the development of work procedures, the design of physical spaces and the work environment, and processes to support organisational learning from experience. ${ }^{3}$

Across health systems, there is an increasing appreciation of the value of HF/E and, slowly, qualified HF/E professionals are employed to work as embedded practitioners alongside clinical teams, even if their number is far behind what is common practice in safety-critical industries (such as aviation, defence and nuclear). ${ }^{4}$ The professionalisation of HF/E in healthcare is still in its infancy, and greater awareness and access to accredited education are required. Here, we present a vision for healthcare HF/E based on the work of the Chartered Institute of Ergonomics and Human Factors (CIEHF), which is the professional body for HF/E in the UK.

\section{HF/E in design}

The fundamental principles of HF/E are related to how the design of equipment, work and workplaces will influence the performance or outcomes of an organisation relative to safety, efficiency and wellbeing. A prospective risk analysis is an approach adopted within other safety critical industries to understand the nature of the threats specific to a particular context or place of work. There is a regulatory mandate for many safety critical industries (eg oil and gas, nuclear and rail) to adopt this approach. HF/E principles are embedded into this process, which enables an organisation to understand and evaluate how the current system design may influence the risk held by the organisation. This form of regulatory mandate does not exist in healthcare, where there are still few resources directed towards the employment of safety or HF/E expertise. ${ }^{5}$

A prospective risk analysis involves a systems perspective; this starts with an understanding of the key healthcare processes: what and who is involved, where is it completed and which 
equipment is used in which environment. This approach can quickly provide an insight into the interactions that are most likely to influence the safety and reliability of the care delivered. Acknowledging the predictable and less predictable scenarios can explore how the system is likely to respond based on the way that work is currently completed, the influence of existing system design and any constraints in resources.

There is a basic need for healthcare to understand the terms of hazard and risk, before prospective risk analysis can become part of safety improvements. ${ }^{5}$ A hazard is anything that has the potential to cause harm. HF/E provides several approaches to identify hazards in technical and sociotechnical systems, including traditional approaches (such as human reliability analysis and the bow-tie method) as well as more recent methods (such as the functional resonance analysis method, which is based on resilience engineering principles). ${ }^{6-8}$ The chance and consequence of a hazard occurring is the risk within a system. These two words provide a vocabulary to develop a shared understanding at any level of a healthcare organisation. They can assist in communicating, prioritising and managing the risks presented within a particular healthcare environment, process or context. This helps reduce the need for patient-facing staff having to be reactive in constantly managing and reporting similar risks, and supports the development of a proactive management strategy that is owned at the appropriate level of an organisation.

In healthcare, clinical autonomy and professional accountability are often relied upon to manage all natures of systemic risks. The sustainability of a workforce and their ability to perform reliably and consistently is intrinsically linked to the context where work occurs. $\mathrm{HF} /$ E principles fully integrated within an organisation can help identify and manage the operational risk associated to the design of working conditions to optimise the performance of clinicians. Healthcare is yet to apply systematically and rigorously the evidence and science that HF/E applies in other industries (Box 1). The principles behind an HF/E approach recognise that the safety, performance and wellbeing of clinical staff are intrinsically linked to patient safety and the performance and efficiency of a healthcare organisation.

\section{HF/E and digital transformation}

The introduction of digital technologies is often regarded as essential for meeting future challenges. ${ }^{10}$ This applies especially

Box 1. Fatigue key performance indicators in the rail industry

The rail industry recognises that staff fatigue can contribute to risk. Rail operators adopt fatigue risk management systems to inform how their organisation can design and monitor shift patterns and working conditions to minimise fatigue, stress and burnout.

The Office of Rail and Road suggests examples of key performance indicators for organisations to consider to indicate the presence of fatigue-inducing factors related to shift design, such as:

percentage of shifts greater than 8 hours

$>$ cumulative hours worked

$>$ duration of rest days

cumulative time awake. ${ }^{9}$ to the use of AI applications, which can exploit the exponentially growing amount of routinely collected data that cannot be analysed anymore by existing teams of human analysts. There is no shortage of examples of the potential benefit that AI can bring to health services, including deep learning applications to support the interpretation of radiological images and AI-powered patientfacing symptom checkers and chatbots. ${ }^{11}$

Evaluation studies of a number of AI algorithms have produced encouraging results, with many studies suggesting that the performance of the AI was at least as good as that of human experts; for example, in the detection of skin cancer or the detection of diabetic retinopathy. ${ }^{12,13}$ However, looking across these studies, the focus of the evaluation is usually on the performance of the AI on a narrowly defined task. The evaluation is typically undertaken by the technology developers, and independent evaluation remains the exception. The number of human participants tends to be small, and prospective trials are still infrequent. As a result, the evidence base to date about the actual performance of AI in real-world clinical settings remains weak. ${ }^{14}$ It is likely that the real challenges for the adoption of AI will arise when algorithms are integrated into clinical systems to deliver a service in collaboration with clinicians as well as other technology. It is at this clinical system level, where teams consisting of healthcare professionals and AI systems cooperate and collaborate to provide a service, that HF/E challenges will come to the fore. ${ }^{15}$

Taking a systems perspective can help designers and users of digital technologies to anticipate and understand the impact of using digital technologies in real-world settings (Box 2).

Box 2. Impact of using artificial intelligence for detecting diabetic retinopathy in a real-world

\section{setting}

A Google research team developed a deep learning artificial intelligence (AI) system to detect referrable diabetic retinopathy from fundus images. A retrospective evaluation based on data from over 25,000 images of diabetic patients in Thailand suggested that the AI system had significantly higher sensitivity and slightly lower specificity compared with a panel of 13 human graders. ${ }^{16}$ A subsequent qualitative study by Google researchers evaluated the real-world impact of the algorithm as deployed in 11 clinics across Thailand. ${ }^{17}$ The findings of this study highlight many of the human factors and ergonomics aspects that emerge only once the AI is embedded in the real world, including:

$>$ a high degree of variation in the process of eye screening across different clinics

$>$ variability regarding the physical environment including light conditions affecting the quality of fundus photos

a high rejection rate of images by the AI system due to inadequate image quality even though human readers were able to screen the images

additional workload of staff as they were trying to retake photos several times to ensure suitable image quality (for the AI), also resulting in increased patient waiting times

$>$ increased numbers of non-essential referrals due to the inability of the AI to process lower-quality images causing frustration among patients who were required to travel large distances as a result

> significant backlogs during periods where the internet speed dropped, causing delays, patient frustration and increased stress levels among staff. 
Examples include the impact on situation awareness, clinician workload, the potential for overreliance on technology, the need for trust and the role of explanation (ie explainable AI), and training requirements of clinicians and patients for using these technologies.

\section{$\mathrm{HF} / \mathrm{E}$ to support organisational learning}

The NHS and health systems worldwide are aiming to become systems dedicated to learning, but the focus is still quite narrowly on learning from harm, and too often recommendations focus on education, training and disciplining. ${ }^{18} \mathrm{HF} / \mathrm{E}$ promotes a systems approach to organisational learning that can inform and transform how we learn from experience. The CIEHF guidance Achieving sustainable change outlines an integrated framework for organisational learning (Fig 1) that appreciates the complexity and pressures of modern healthcare organisations, which is particularly relevant during the current COVID-19 pandemic where organisations and health systems need to learn about how to navigate a complex and dynamic situation full of uncertainty. ${ }^{19}$

The guidance frames organisational learning in terms of both the organisational mindset and the actions or the process to implement it. The mindset is about how an organisation approaches organisational learning. The guidance contains prompts to encourage organisations to think about their learning goals: who is involved in organisational learning, how deep their learning is, the types of situations they try to learn from and the processes they have in place to foster learning. The actions describe how organisational learning actually takes place in

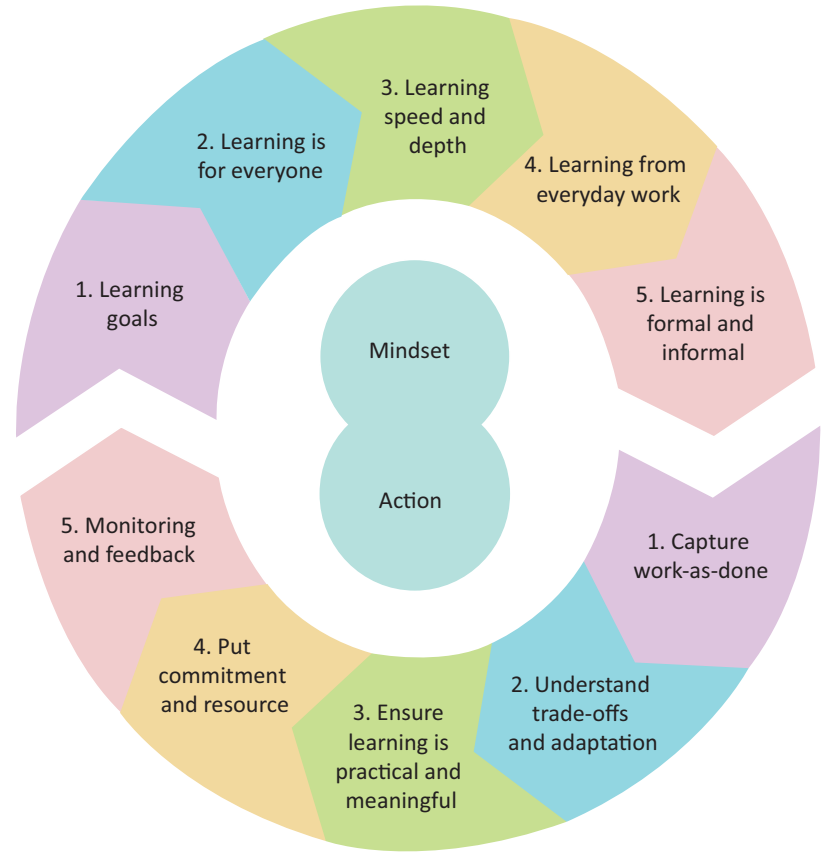

Fig 1. The Chartered Institute of Ergonomics and Human Factors organisational learning framework for achieving sustainable change. Adapted with permission from CIEHF. an organisation, or how it is carried out. The guidance puts emphasis on learning from everyday work (rather than just from incidents) in order to understand how people adapt to situations and changes; it emphasises that staff should have an active role to play in organisational learning in order to ensure that learning is meaningfully related to practice; and, where possible, staff should be encouraged to take ownership for taking changes forward, and should be given authority and resource to do so. Finally, any changes that are implemented will likely require further adjustments over time and, therefore, the learning process should be continuous and feedback from staff should be sought and given.

Based on the HF/E perspective, the intention is to move away from negative notions of incidents, errors and blame; to encourage organisations to reflect on what goes well even when situations are challenging; and explore how safe spaces (in terms of psychological safety) can be created where people can contribute to organisational learning and where they can take ownership of change and improvement. ${ }^{20}$

\section{HF/E during COVID-19}

The COVID-19 pandemic provided HF/E specialists and professional bodies (such as CIEHF) with significant opportunities to collaborate with senior healthcare leaders and clinicians to contribute much-needed systems and user-centred design thinking and methods to support pressing national and international challenges. HF/E specialist input was of great benefit in areas where major challenges were and are being faced.

The safety and usability of deploying rapidly manufactured ventilators within temporary care settings

In the early period of the pandemic, it became quickly evident that there was likely to be massively increased demand internationally for mechanical ventilators to treat COVID-19 patients in intensive care units (ICUs) in converted hospital wards and newly designed field hospitals. Given the anticipated numbers of patients who would require admission to ICUs in the NHS (and in global health systems) at the time, it was apparent that traditional manufacturers of this vital life-saving medical technology would be unable to cope unaided with the unprecedented demand, and that additional equipment suppliers would be required. The UK government, in direct response, publicly called for new manufacturers to volunteer to increase the scale of ventilator production through a process of rapidly manufactured ventilator systems (RMVS). In recognising some of the design, usability and safety challenges that are evident with many medical devices and would highly likely be apparent, especially for novel manufacturers, the CIEHF rapidly assembled a project team of chartered HF/E specialists and senior clinicians to provide fundamental RMVS design guidance. ${ }^{21}$ The goal was to support RMVS manufacturers with a structured and accessible process for the design of the user interface, instructions for use and training, based on four key stages: identification of users, description of the environment, task analysis and risk assessment (Fig 2). Fortunately, the anticipated demand for additional ventilator capacity did not materialise, and this rapidly developed guidance was not 

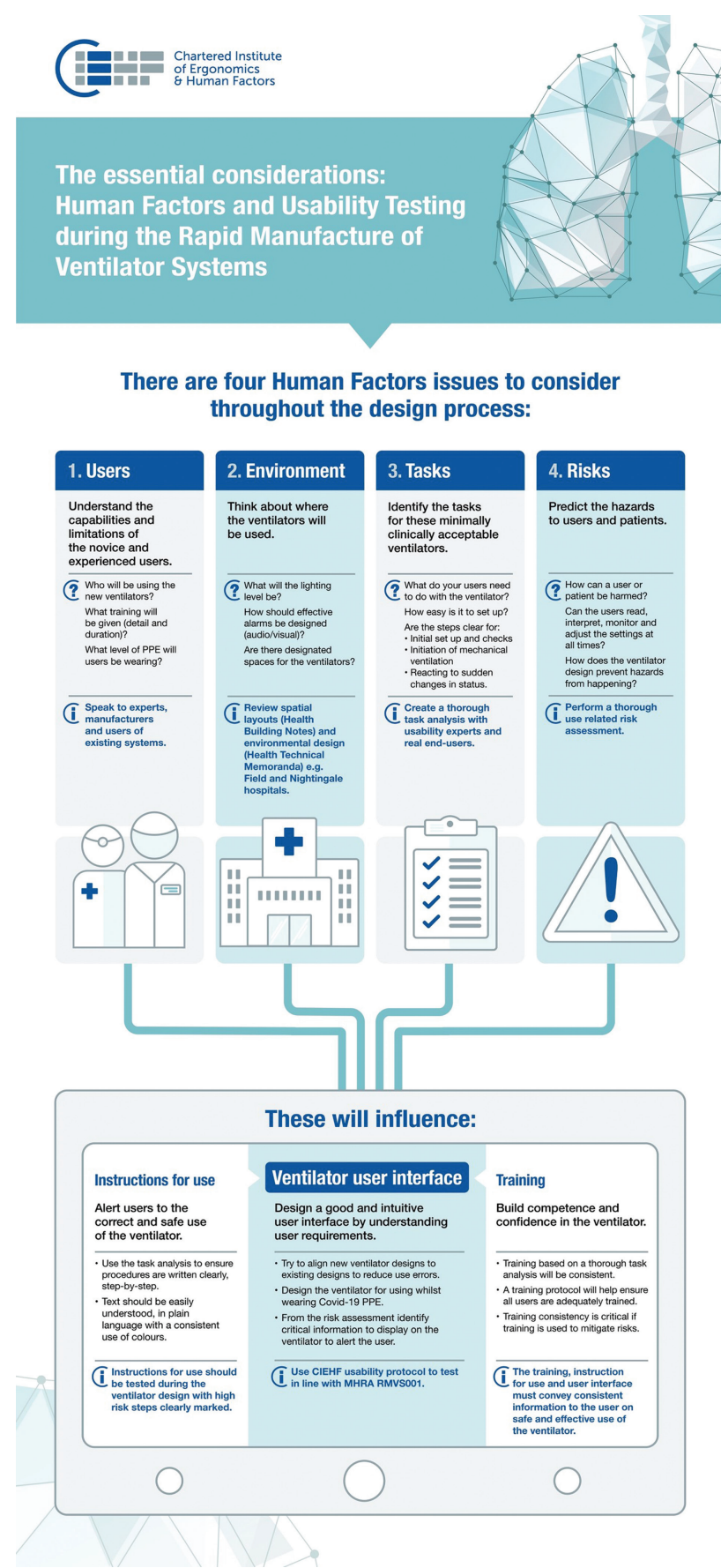

Fig 2. The Chartered Institute of Ergonomics and Human Factors design guidance for rapidly manufactured ventilator systems. Reproduced with permission from CIEHF.

required at that time, but it still stands as a testimony to the role of HF/E and the response of CIEHF in a national crisis.

\section{Setting up national vaccination programmes}

In related improvement work about national COVID-19 vaccination programmes, HF/E specialists reviewed and analysed the design and operation of a small number of healthcare vaccination site facilities in Scotland and undertook workshops with vaccinators, site leaders and support staff. While many positive design features and risk control measures were found to be in place, additional actions for improvement where made. It was agreed that the sharing of this important learning would benefit national and international vaccination programmes given that related immunisation progress was variable globally. For example, recommendations included:

> approaches for understanding the target population to reflect the needs and capabilities of all stakeholders, including people in remote and rural areas

$>$ suggestions for well-designed and usable tools and technologies to support performance, including scheduling tools to ensure people can book and change appropriate appointments easily

> consideration of the physical environment such as sufficient space for social distancing including use of stairs and lifts

$>$ agile education and training that can keep pace with the fast rate of adaptation and change within vaccination programmes.

Key HF/E lessons for stakeholders involved in setting up national vaccination programmes were summarised in the CIEHF guidance Vaccinating a nation. ${ }^{22}$ The guidance sets out $10 \mathrm{HF} / \mathrm{E}$ principles to support the safe roll-out of national vaccination efforts. These principles (Fig 3) are based on a systems approach and guide stakeholders through the stages of identifying salient characteristics of the work systems, improving designs, work instructions and training, and continuously monitoring and adapting to learn from experience.

\section{Embedding HF/E in healthcare practice}

Moving forward, there is need for more rigorous and science-based $\mathrm{HF} / \mathrm{E}$ contribution to healthcare design and delivery. There are two aspects that will be crucial enablers.

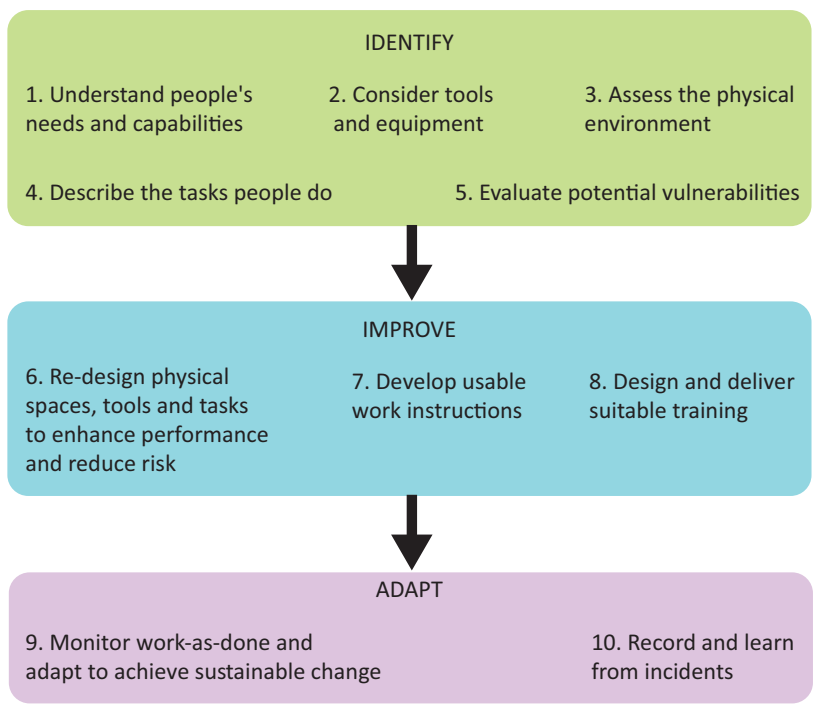

Fig 3. Ten human factors and ergonomics principles to support the setup of national vaccination programmes. Adapted with permission from CIEHF. 
Enhancing and professionalising HF/E knowledge among healthcare professionals with active roles in quality improvement and patient safety

Until very recently, there was no formal career structure for HF/E healthcare professionals. Now, CIEHF has launched a 'healthcare human factors learning pathway' to support both academic (postgraduate certificate) and learning-at-work routes to achieve the status of technical specialist or TechCIEHF (Healthcare). This professional approach can support healthcare professionals and patient safety specialists to effectively apply HF/E theories and approaches in clinical practice.

\section{Having embedded suitably qualified (CErgHF) HF/E practitioners}

In the last few years, hospitals across the USA have begun to employ clinically embedded HF/E professionals, where fully qualified HF/E practitioners work alongside clinicians every day to apply HF/E methods and perspectives. ${ }^{4}$ Healthcare organisations need to know how they can both employ HF/E specialists and also train key staff members through accredited routes. Embedding suitably qualified HF/E professionals within a healthcare organisation enables the HF/E practitioner to understand an establishment's structure, culture and ways of working. Through their work, the HF/E practitioner is able to build relations with colleagues to become an established member of the team enabling the opportunity for timely HF/E input. By working alongside all levels and groups of staff, familiarity and trust is gained allowing a multidisciplinary approach to be taken, which will likely lead to the implementation of more effective and sustainable solutions. HF/E professionals who are embedded within an organisation have the ability to instigate and sustain conversations regarding the discipline through a variety of means such as working alongside other staff groups, the production of reports, attendance at meetings, formal presentations and the more informal 'corridor conversations'. Through this, the knowledge and understanding of HF/E, along with its importance and relevance within healthcare, will develop. Pairing the knowledge and skills of a qualified HF/E professional embedded in an organisation with accredited HF/E education available to other key staff members will further advance the application of HF/E, benefiting human wellbeing and system performance.

Clinicians leading improvement and service design should work with specialists and receive development in $\mathrm{HF} / \mathrm{E}$ beyond team-working and non-technical skills. HF/E should be consistently applied to proactive design of care systems to maximise safety and wellbeing.

\section{Acknowledgements}

We are grateful to CIEHF members and volunteers who have contributed to the development of HF/E guidance during the COVID-19 pandemic.

\section{Conflicts of interest}

Noorzaman Rashid is chief executive of the CIEHF. Mark Sujan, Laura Pickup, Paul Bowie, Sue Hignett, Fran Ives and Helen Vosper are registered members of the CIEHF.

\section{References}

1 Carayon P, Hundt AS, Karsh B et al. Work system design for patient safety: the SEIPS model. Qual Saf Health Care 2006;15(suppl 1): i50-8.

2 Catchpole K. Spreading human factors expertise in healthcare: untangling the knots in people and systems. BMJ Qual Saf 2013;22:793-7.

3 Holden RJ, Carayon P, Gurses AP et al. SEIPS 2.0: a human factors framework for studying and improving the work of healthcare professionals and patients. Ergonomics 2013;56:1669-86.

4 Catchpole K, Bowie P, Fouquet S, Rivera J, Hignett S. Frontiers in human factors: embedding specialists in multi-disciplinary efforts to improve healthcare. Int J Qual Health Care 2021;33(Suppl 1): 13-8.

5 Sujan MA, Habli I, Kelly TP, Gühnemann A, Pozzi S, Johnson CW. How can health care organisations make and justify decisions about risk reduction? Lessons from a cross-industry review and a health care stakeholder consensus development process. Reliability Engineering \& System Safety 2017;161:1-11.

6 Sujan MA, Embrey D, Huang H. On the application of Human Reliability Analysis in healthcare: Opportunities and challenges. Reliability Engineering \& System Safety 2020;194:106189.

7 McLeod RW, Bowie P. Bowtie Analysis as a prospective risk assessment technique in primary healthcare. Policy and Practice in Health and Safety 2018;16:177-93.

8 Hollnagel E. FRAM, the functional resonance analysis method: modelling complex socio-technical systems. Ashgate, 2012.

9 Office of Rail and Road. Fatigue - key performance indicators. ORR, 2017.

10 Peek N, Sujan M, Scott P. Digital health and care in pandemic times: impact of COVID-19. BMJ Health Care Inform 2020; 27:e100166.

11 Topol EJ. High-performance medicine: the convergence of human and artificial intelligence. Nature Medicine 2019;25:44-56.

12 Esteva A, Kuprel B, Novoa RA et al. Dermatologist-level classification of skin cancer with deep neural networks. Nature 2017:542:115.

13 Gulshan V, Peng L, Coram M et al. Development and validation of a deep learning algorithm for detection of diabetic retinopathy in retinal fundus photographs. JAMA 2016;316:2402-10.

14 Nagendran M, Chen Y, Lovejoy CA et al. Artificial intelligence versus clinicians: systematic review of design, reporting standards, and claims of deep learning studies. BMJ 2020;368:m689.

15 Sujan M, Furniss D, Grundy K et al. Human factors challenges for the safe use of artificial intelligence in patient care. BMJ Health Care Inform 2019;26:e100081.

16 Ruamviboonsuk P, Krause J, Chotcomwongse P et al. Deep learning versus human graders for classifying diabetic retinopathy severity in a nationwide screening program. npj digital medicine 2019; 2:25.

17 Beede E, Baylor E, Hersch F et al. A human-centered evaluation of a deep learning system deployed in clinics for the detection of diabetic retinopathy. Proceedings of the $2020 \mathrm{CHI}$ Conference on Human Factors in Computing Systems 2020: $1-12$.

18 Sujan M. An organisation without a memory: A qualitative study of hospital staff perceptions on reporting and organisational learning for patient safety. Reliability Engineering \& System Safety 2015;144:45-52.

19 Sujan M, Bowie P, Smyth M, Rashid N. Achieving sustainable change: Capturing lessons from COVID-19. Chartered Institute of Ergonomics and Human Factors, 2020.

20 Sujan MA, Huang $\mathrm{H}$, Braithwaite J. Learning from incidents in health care: critique from a safety-II perspective. Safety Science 2017;99:115-21. 
21 Hignett S, Edmonds J, Herlihey T et al. Human factors/ergonomics to support the design and testing of rapidly manufactured ventilators in the UK during the COVID-19 pandemic. Int J Qual Health Care 2021;33:4-10.

22 Sujan M, Hignett S, Rashid N. Vaccinating a nation: ten human factors and ergonomics principles. Chartered Institute of Ergonomics and Human Factors, 2021.
23 Hignett S, Bowie P. Why is the NHS still harming patients? Taking a professional approach to patient safety for COVID-19 and beyond. Science in Parliament 2020:76:14-6.

Address for correspondence: Dr Mark Sujan, Human Factors Everywhere, 28 Cavell Way, Woking GU21 2TJ, UK.

Email: mark.sujan@gmail.com

Twitter: @MarkSujan 